\title{
Dopant-Induced Ignition of Helium Nanodroplets in Intense Few-Cycle Laser Pulses
}

\author{
S. R. Krishnan, ${ }^{1}$ L. Fechner, ${ }^{2}$ M. Kremer, ${ }^{1}$ V. Sharma, ${ }^{1}$ B. Fischer, ${ }^{1}$ N. Camus, ${ }^{1}$ J. Jha, ${ }^{3}$ M. Krishnamurthy, ${ }^{3}$ T. Pfeifer, ${ }^{1}$ \\ R. Moshammer, ${ }^{1,4}$ J. Ullrich, ${ }^{1,4}$ F. Stienkemeier, ${ }^{2}$ M. Mudrich, ${ }^{2, *}$ A. Mikaberidze, ${ }^{5}$ U. Saalmann, ${ }^{4,5}$ and J.-M. Rost ${ }^{4,5}$ \\ ${ }^{1}$ Max-Planck-Institut für Kernphysik, Saupfercheckweg 1, 69117 Heidelberg, Germany \\ ${ }^{2}$ Physikalisches Institut, Universität Freiburg, Hermann-Herder-Straße 3, 79104 Freiburg, Germany \\ ${ }^{3}$ Tata Institute of Fundamental Research, 1 Homi Bhabha Road, Mumbai 400005, India \\ ${ }^{4}$ Max Planck Advanced Study Group at CFEL, Luruper Chaussee 149, 22761 Hamburg, Germany \\ ${ }^{5}$ Max-Planck-Institut für Physik komplexer Systeme, Nöthnitzer Straße 38, 01187 Dresden, Germany
}

(Received 22 July 2011; published 19 October 2011)

\begin{abstract}
We demonstrate ultrafast resonant energy absorption of rare-gas doped He nanodroplets from intense few-cycle ( $10 \mathrm{fs}$ ) laser pulses. We find that less than 10 dopant atoms "ignite" the droplet to generate a nonspherical electronic nanoplasma resulting ultimately in complete ionization and disintegration of all atoms, although the pristine He droplet is transparent for the laser intensities applied. Our calculations at those intensities reveal that the minimal pulse length required for ignition is about $9 \mathrm{fs}$.
\end{abstract}

DOI: 10.1103/PhysRevLett.107.173402

PACS numbers: 36.40.Gk, 31.70.Hq, 52.50.Jm, 87.15.ht

The ionization dynamics of atomic clusters in intense ultrashort laser pulses has been an active area of research in recent years at near-infrared (NIR), vacuum-ultraviolet, and soft x-ray wavelengths [1-3]. The energy absorption of NIR light by rare-gas clusters is far more efficient as compared to atomic jets or planar solid targets when irradiated with similar laser pulses. The underlying mechanism is resonance absorption by an electronic nanoplasma which forms through illumination with the light $[4,5]$. The resonance occurs when the background ions of total charge $Q$ occupy a large enough sphere of radius $R$ such that the nanoplasma eigenfrequency $\Omega=\sqrt{Q / R^{3}}$ matches the frequency $\omega$ of the laser light [1]. The resonance condition $\Omega=\omega$ is achieved on the time scale of atomic motion (sub- or a few picoseconds) since the cluster, i.e., the ions, must expand in order for the ions to become sufficiently dilute. This has been verified in experiments where pumpprobe delay [6] or pulse durations are varied [7], as well as in calculations [5].

The fact that this resonance absorption at NIR frequencies relies predominantly on atomic expansion has two consequences: First, it requires time scales of ionic motion in spite of its electronic nature; second, it does not occur in isolated atoms or those inside the bulk in condensed phase. One may ask if there is another possibility for this resonant coupling of energy from the light pulse to the electrons which is not only extremely efficient but also fast, bypassing atomic expansion. Indeed, this can be achieved as theoretically proposed and demonstrated [8], if one uses a two-component system with a few seed atoms with ionization potentials lower than the majority of atoms which belong to the other component. If the spherical symmetry of the system is suitably broken (e.g., Xe atoms in the center of a He droplet irradiated by linearly polarized light), a "cigar-shaped" nanoplasma is formed. It has two eigenfrequencies which bracket the eigenfrequency of a spherical plasma. The lower one (along the linear laser polarization) is resonant with $790 \mathrm{~nm}$ laser light right away at typical atomic cluster densities leading to almost immediate resonance absorption without the need for atomic expansion. This not only allows us to realize the efficient energy transfer very quickly, now limited essentially by the rise time of the laser pulse, but also opens the resonant light absorption to other forms of matter which do not (Coulomb) explode.

Here, we report on experiments which demonstrate this purely electronic resonance absorption with rare-gas doped He droplets illuminated by a few-cycle pulse of a duration as short as $10 \mathrm{fs}$ at $790 \mathrm{~nm}$. This rules out the influence of any kind of atomic motion. Reducing the pulse length even further must eventually prevent even this electronic resonance absorption. Indeed, as we will demonstrate with calculations, there is a clear transition from dopantinduced resonance absorption to a regime dominated by static field ionization [9] if the pulse length falls below a critical value which depends, of course, on the intensity of the laser pulse.

He nanodroplets can be doped with a well-controlled number of rare-gas atoms which aggregate at the center of the droplet $[10,11]$. These are exceptionally suitable to demonstrate the role of seed atoms in resonance absorption, since the laser intensity can be chosen such that the light couples almost exclusively to the dopants whereas the pristine He droplet is transparent. Interestingly, this applies to NIR $[8,12]$ as well as to $x$-ray [9] frequencies. Despite its transparency, the droplet becomes highly active once the core of seed atoms is ionized.

In the experiment, a beam of He nanodroplets is produced by expanding pressurized ${ }^{4} \mathrm{He}$ gas $(70-90$ bar) through a nozzle $(5 \mu \mathrm{m}$ in diameter) maintained at a temperature of $15-25 \mathrm{~K}$. By varying the nozzle temperature in this range, the mean number of $\mathrm{He}$ atoms per droplet 
is adjusted in the range $10^{3}-10^{5}$. Owing to their low internal temperature $\sim 370 \mathrm{mK}$, these droplets are in a superfluid state $[10,11]$. They are doped by passing the skimmed beam of pure droplets through a cylindrical doping cell that is $3 \mathrm{~cm}$ long with collinear apertures $(\varnothing=3 \mathrm{~mm})$. The mean number of dopants per nanodroplet $K$ is regulated through controlled leaking of the desired rare gas into this cell using a dosing valve (leak rate $<10^{-10} \mathrm{mbar} \mathrm{l} / \mathrm{s}$ ). The pressure in the doping cell is monitored by a directly attached vacuum gauge.

Recently, we have simulated the pickup process of alkali atoms that form high-spin clusters by using a Monte Carlo model [13]. Here, we use the same model adapted to raregas dopants to validate the estimate of doping levels and to estimate the loss of He due to evaporation caused by the doping process: The number $K$ of dopant atoms is ascertained from the cell pressure and the droplet size according to the modified Poissonian pickup statistics [11] using the semiempirical formula of Kuma et al. [14], which takes evaporation of $\mathrm{He}$ atoms into account.

Intense few-cycle laser pulses $(\sim 10 \mathrm{fs})$ at a central wavelength of $790 \mathrm{~nm}$ with peak intensities in the range $10^{14}-10^{15} \mathrm{~W} / \mathrm{cm}^{2}$ are generated from a Ti-sapphire based mode-locked laser system. Photoions are detected by a time-of-flight (TOF) spectrometer in the Wiley-McLaren geometry. TOF mass spectra of $\mathrm{He}^{++}$ions for different mean doping numbers $K$ of $\mathrm{Xe}$ in a droplet containing $1.5 \times 10^{4} \mathrm{He}$ atoms are shown in the inset in Fig. 1(b). The $\mathrm{He}^{++}$peak, which is characteristic for ionizing doped $\mathrm{He}$ droplets and is absent in mass spectra of atomic He gas, is split as a result of the finite angular acceptance of the spectrometer into two components due to ions with high

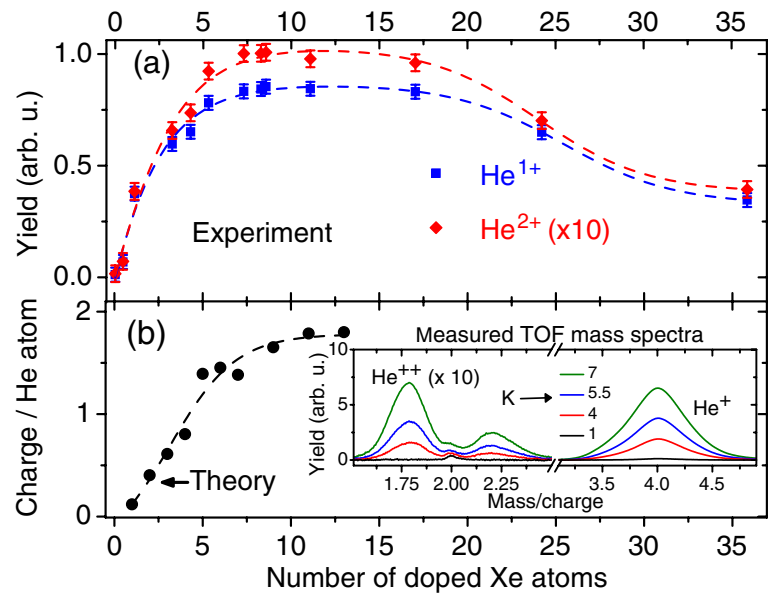

FIG. 1 (color online). (a) Yields of $\mathrm{He}^{++}$and $\mathrm{He}^{+}$ions as a function of the mean number of Xe dopants in a nanodroplet containing $1.5 \times 10^{4} \mathrm{He}$ atoms at a peak laser intensity of $7 \times 10^{14} \mathrm{~W} / \mathrm{cm}^{2}$. (b) Numerical calculation of the charge per $\mathrm{He}$ atom as a function of the number of Xe atoms in a droplet containing $4000 \mathrm{He}$ atoms. All lines are to guide the eye. Inset: Time-of-flight mass spectra of $\mathrm{He}^{++}$and $\mathrm{He}^{+}$ions for different numbers of dopant Xe atoms $(K)$ as shown in the legend. momenta directed towards and away from the detector, respectively. The yield of $\mathrm{He}^{++}$and $\mathrm{He}^{+}$ions is extracted from the TOF mass spectra by integrating over the respective mass peaks.

Figure 1(a) presents the doping dependence of the yields of $\mathrm{He}^{++}$and $\mathrm{He}^{+}$at a peak intensity of $7 \times 10^{14} \mathrm{~W} / \mathrm{cm}^{2}$ of the laser pulse. A gradual increase of doping number $K=1$ up to 10 leads to a dramatic steplike increase in the yields of $\mathrm{He}^{+}$and $\mathrm{He}^{++}$ions. We refer to this nanoplasma formation as dopant-induced ignition (DII). The saturation of ion yields, and equivalently the buildup of charge in the nanoplasma, occurs for a critical doping number $K_{\text {cr }}$ of just $\sim 7( \pm 1.4)$. The ionization of the He droplet is not enhanced any further by adding more dopants. Based on classical molecular dynamics [5], we have tailored a theoretical description to the present problem of doped $\mathrm{He}$ droplets (for details of the approach, see $[8,12]$ ). The result from our calculation shows [Fig. 1(b)] qualitatively the same behavior, although a direct comparison of the charge to yields is difficult since the experiment is an average over the droplet size and doping distributions as well as over intensities in the focal volume. Moreover, in the theoretical result, electron recombination [15] is not taken into account. Yet, the curves agree well qualitatively and even quantitatively regarding the critical dopant number at which the saturation of charging occurs. This agreement points to a robust underlying mechanism, in which the number of ions after recombination is directly related to energy absorption during the pulse.

The critical doping number is remarkably independent of the mean droplet size as shown in Fig. 2 having the value of $10( \pm 2)$ for mean droplet sizes of 4500 and 15000 atoms

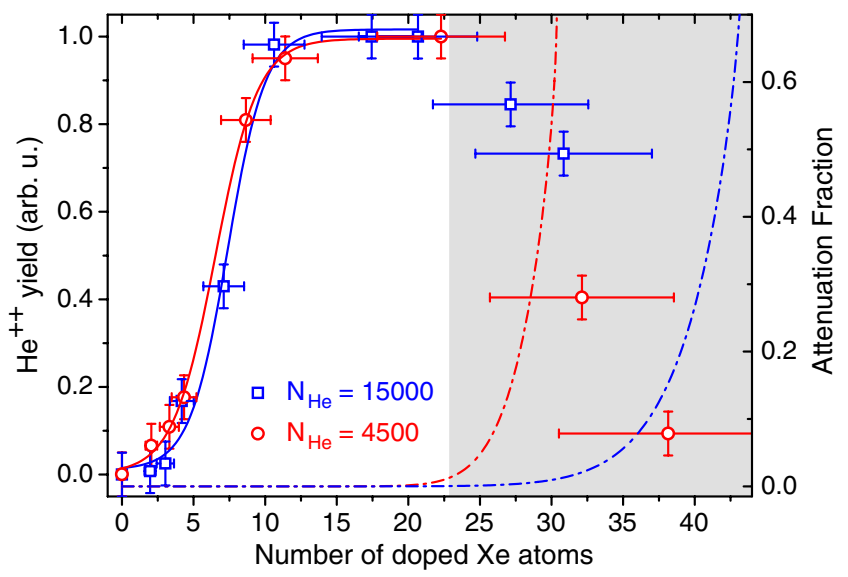

FIG. 2 (color online). Xe doping dependence of $\mathrm{He}^{++}$yields at a peak laser intensity of $1.5 \times 10^{14} \mathrm{~W} / \mathrm{cm}^{2}$ for droplets with a mean number of $\mathrm{He}$ atoms per droplet $N_{\mathrm{He}}=4500$ (red) and $N_{\mathrm{He}}=15000$ (blue). Horizontal bars present the error in estimating the number of doped Xe atoms. The dash-dotted curves present the fraction of droplets lost due to complete evaporation, which is significant only for numbers of Xe dopant atoms $K \gtrsim 20$ (gray shading). 
at a peak laser intensity of $1.5 \times 10^{14} \mathrm{~W} / \mathrm{cm}^{2}$. The error in estimating the number of dopants using Kuma's approach [14] is also shown. The decrease of doubly charged He for increasing $K$ in Figs. 1-3 is related to the doping process: When dopant atoms are picked up sequentially and aggregate inside the droplet, collisional as well as binding energy is released into the droplet. This leads to an increasing fraction of $\mathrm{He}$ droplets that completely evaporate at high doping pressure. The corresponding estimate from the Monte Carlo simulation for Xe doping is indicated in Fig. 2 as dashed lines. Clearly, the onset of significant nanodroplet destruction in this case of Xe doping occurs well beyond $K_{\mathrm{cr}} \sim 10$ observed in the experiment. Thus, the process of DII is not influenced by evaporation effects.

The insensitivity of DII with respect to the droplet sizes results simply from the fact that DII sets in with the formation of a cigar-shaped plasma which is much smaller than the droplet itself [8]. In contrast, DII should depend on the laser intensity and similarly on the ionization potential of the seed atoms, as well as on the pulse length. The former dependences are illustrated with experimental results in Fig. 3. As expected, $K_{\text {cr }}$ is smaller for higher intensities and/or lower ionization potentials.

We determined numerically the average $\mathrm{He}$ charge in a droplet $Y(K)$ as a function of the number of Xe dopants $K$. One can see in Fig. 4 that at the intensity of $7 \times 10^{14} \mathrm{~W} / \mathrm{cm}^{2}$ a qualitative change of the average charge per He atom $Y(K)$ occurs between 7 and 9 fs pulse length: For longer pulses, DII (solid lines) is operative with a fast rise followed by saturation at full ionization of the $\mathrm{He}$ atoms $(Y=2)$. For shorter pulses, the average charge shows a gradual rise $Y \propto K^{3 / 2}$ (dashed lines), which is characteristic for ionization due to the static electric field of the laser ionized $\mathrm{Xe}$ ions in the center of the droplet.

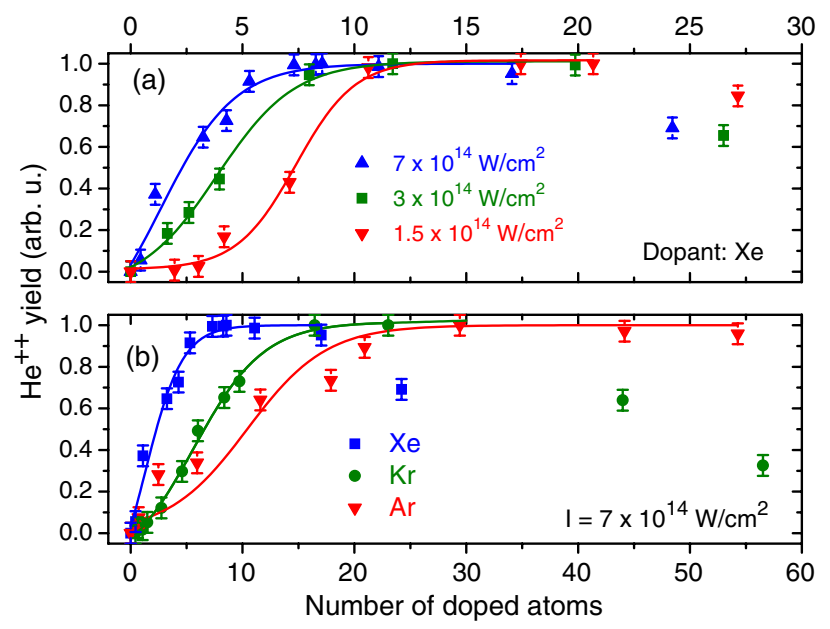

FIG. 3 (color online). The $\mathrm{He}^{++}$yields for a droplet containing $15000 \mathrm{He}$ atoms as a function of the number of Xe dopant atoms (a) for different laser intensities $I$ and (b) for different dopant species at $I=7 \times 10^{14} \mathrm{~W} / \mathrm{cm}^{2}$.
To derive this dependence, we assume that all Xe ions constitute a point charge at the center of the droplet and that the density of He atoms is uniform within the spherical droplet. Then the problem is of purely radial nature, and a sphere exists which contains only doubly ionized $\mathrm{He}$ atoms. Its radius is given by $R_{++}=\sqrt{q_{\mathrm{Xe}} K / E_{\mathrm{bs} 2}}$, where $q_{\mathrm{Xe}}$ is the average charge of each of the $K$ dopant atoms and $E_{\mathrm{bs} 2}$ is the electric field necessary to ionize the second electron in He through barrier suppression (Bethe rule) $[9,16]$. The number of $\mathrm{He}$ atoms inside this sphere is given by $\quad N_{++}=4 \pi \rho_{\mathrm{He}} R_{++}^{3} / 3=4 \pi \rho_{\mathrm{He}}\left(q_{\mathrm{Xe}} K / E_{\mathrm{bs} 2}\right)^{3 / 2} / 3$, where $\rho_{\mathrm{He}}$ is the density of He in the droplet. We find the number of singly ionized $\mathrm{He}$ atoms $N_{+}$in a similar way and obtain the average ion charge per He atom by using $Y=\left(N_{+}+2 N_{++}\right) / N_{\mathrm{He}}$, where $N_{\mathrm{He}}$ is the total number of $\mathrm{He}$ atoms in the droplet. This yields

$$
Y(K)=\frac{4 \pi \rho_{\mathrm{He}}}{3 N_{\mathrm{He}}}\left(E_{\mathrm{bs} 1}^{-3 / 2}+E_{\mathrm{bs} 2}^{-3 / 2}\right) q_{\mathrm{Xe}}^{3 / 2} K^{3 / 2},
$$

where $E_{\mathrm{bs} 1}$ and $E_{\mathrm{bs} 2}$ are the first and the second barrier suppression fields for $\mathrm{He}$, respectively, much larger than the laser field, which can be safely neglected for this consideration. Assuming that $q_{\mathrm{Xe}}$ does not depend strongly on the number of Xe atoms $K$ (this is fulfilled very well for $K$ between 2 and 15 as seen from the molecular dynamics simulations), we conclude that $Y \propto K^{3 / 2}$; i.e., the static field ionization due to positively charged dopants follows a characteristic power law.

Although it is clear from Fig. 4 that for increasing pulse lengths the average charge per He atom $Y(K)$ is no longer well described by the field ionization power law (dashed lines), a well-defined transition from field ionization to DII for increasing pulse length is difficult to extract. To

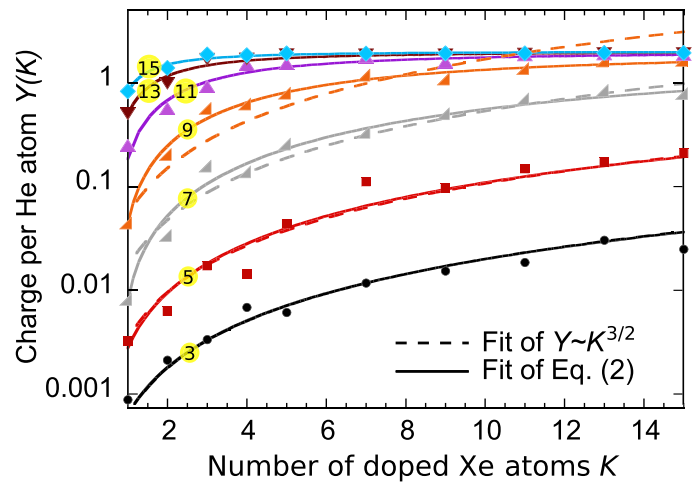

FIG. 4 (color online). Average charge per He atom $Y(K)$ as a function of the number of doped $\mathrm{Xe}$ atoms for a droplet with $4000 \mathrm{He}$ atoms and intensity as in Fig. 1 from molecular dynamics calculations. The curves correspond to different laser pulse lengths (full width at half maximum): from 3 (bottom) to $15 \mathrm{fs}$ (top) in steps of $2 \mathrm{fs}$, as shown in the labels. The lines are fits of the data points according to Eq. (2) (solid line) and with the power law $Y \propto K^{3 / 2}$ corresponding to ionization by a static field for $T \leq 9$ fs (dashed line). For details, see the text. 


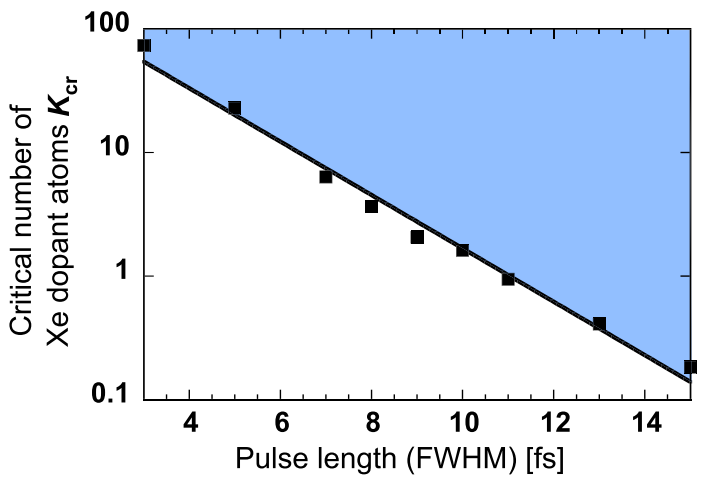

FIG. 5 (color online). The number of dopants for which the average charge per He atom $Y$ in Fig. 4 has a turning point: $Y^{\prime \prime}\left(K_{\mathrm{cr}}\right)=0$. The shaded area distinguishes the regime of DII from the field ionization regime (not shaded), at a peak intensity of $7 \times 10^{14} \mathrm{~W} / \mathrm{cm}^{2}$.

determine the minimal pulse length $T$ for which DII dominates, we parametrize the shape of $Y(K)$ with

$$
Y(K)=Y_{\infty} \frac{(K / \alpha)^{3 / 2}-1}{(K / \alpha)^{3 / 2}+\beta} .
$$

This is the simplest function which contains both limits: For $\beta \gg K / \alpha$, Eq. (2) describes the field ionization behavior $Y(K) \propto K^{3 / 2}$, while for $\beta \ll K / \alpha$, the typical DII shape emerges with a sharp onset followed by saturation at $Y_{\infty}$. The latter is characterized by a negative second derivative $Y^{\prime \prime}(K)$, while for the field ionization power law $Y^{\prime \prime}(K)>0$ holds for all $K$. Hence, $Y^{\prime \prime}\left(K_{\mathrm{cr}}\right)=0$ can be interpreted as the conditional equation for the critical number of dopant atoms where field-ionization-dominated absorption goes over into DII-dominated absorption. Using Eq. (2), we get $K_{\mathrm{cr}}=\alpha(\beta / 5)^{2 / 3}$. The parameters ( $\alpha$ and $\beta$ ) are obtained for each pulse length $T$ by fitting Eq. (2) to the numerically generated data in Fig. $4 . K_{\mathrm{cr}}$ exhibits an exponential dependence on the pulse length $T$, as can be seen from Fig. 5. The shaded area indicates DII with $Y^{\prime \prime}<0$, while below the line of $K_{\mathrm{cr}}$ field ionization rules. Since physically at least one dopant atom is necessary for ionization, DII dominates for $T$ larger than about 9 fs. This is consistent with the qualitative conclusion from Fig. 4 as well as with our experimental result, which demonstrates DII for a pulse length of $10 \mathrm{fs}$.

In summary, we have experimentally demonstrated that it is possible to transfer energy resonantly from a $790 \mathrm{~nm}$ intense few-cycle pulse to bound electrons without the need of atomic expansion of the target. This brings down the time scale of this extremely efficient but relatively slow process from the subpicosecond regime down to a few femtoseconds and, at the same time, allows it to be applied to any form of matter that can be suitably doped with seed atoms. Finally, the DII demonstrated here may also explain the surprising enhancement of light absorption in water doped argon clusters [17].

Stimulating discussions with J. Tiggesbäumker and Th. Fennel and the valuable assistance of O. Bünermann in the Monte Carlo calculations are gratefully acknowledged. This work is supported by the Deutsche Forschungsgemeinschaft.

*marcel.mudrich@physik.uni-freiburg.de

[1] U. Saalmann, C. Siedschlag, and J. M. Rost, J. Phys. B 39, R39 (2006).

[2] T. Fennel et al., Rev. Mod. Phys. 82, 1793 (2010).

[3] H. Wabnitz et al., Nature (London) 420, 482 (2002).

[4] T. Ditmire, T. Donnelly, A. M. Rubenchik, R. W. Falcone, and M. D. Perry, Phys. Rev. A 53, 3379 (1996).

[5] U. Saalmann and J.-M. Rost, Phys. Rev. Lett. 91, 223401 (2003); U. Saalmann, J. Mod. Opt. 53, 173 (2006).

[6] J. Zweiback, T. Ditmire, and M. D. Perry, Phys. Rev. A 59, R3166 (1999).

[7] T. Döppner, T. Fennel, T. Diederich, J. Tiggesbäumker, and K.H. Meiwes-Broer, Phys. Rev. Lett. 94, 013401 (2005).

[8] A. Mikaberidze, U. Saalmann, and J.-M. Rost, Phys. Rev. Lett. 102, 128102 (2009).

[9] C. Gnodtke, U. Saalmann, and J.-M. Rost, Phys. Rev. A 79, 041201 (2009).

[10] J. P. Toennies and A. F. Vilesov, Angew. Chem., Int. Ed. 43, 2622 (2004).

[11] F. Stienkemeier and K. Lehmann, J. Phys. B 39, R127 (2006).

[12] A. Mikaberidze, U. Saalmann, and J. M. Rost, Phys. Rev. A 77, 041201 (2008).

[13] O. Bünermann and F. Stienkemeier, Eur. Phys. J. D 61, 645 (2011).

[14] S. Kuma, H. Goto, M. N. Slipchenko, A. F. Vilesov, A. Khramov, and T. Momose, J. Chem. Phys. 127, 214301 (2007).

[15] C. Peltz and T. Fennel, Eur. Phys. J. D 63, 281 (2011).

[16] H. A. Bethe and E. Salpeter, Quantum Mechanics of Oneand Two-Electron Atoms (Springer, Berlin, 1957).

[17] J. Jha and M. Krishnamurthy, J. Phys. B 41, 041002 (2008). 